\section{A CLINICAL LECTURE ON}

\section{THE SIGNIFICANCE OF DILATATION OR GASTRECTASIA IN FUNCTIONAL AND ORGANIC DISEASES OF THE STOMACH.}

BY M. A. BOYD, M.D. Physician, Mater Misericordiæ Hospital, Dublin.

GASTRIC dilatation or gastrectasia in one or other of its forms is so frequently present in almost all organic or functional affections of the stomach, and its existence is so frequently overlooked by us in hasty examinations of that organ that I may be pardoned for going into details of the various affections in which this symptom is met with. I may say at the outset dilatation of the stomach is not a disease, but only a symptom of disease.

Gastric dilatation presents itself in two forms : complete dilatation and incomplete or partial dilatation.

In complete dilatation all the coats of the entire stomach are stretched, and its cavity enlarged to twice or perhaps thrice its original size, the organ extending across the abdomen from one hypochondriac region to the other, or when the stomach is dislocated downwards by the weight of its own fluid contents and the yielding of the gastro-hepatic ligaments, we may find it occupying the entire of the lower portion of the abdominal cavity, extending from one iliac region to the other.

In the second form-incomplete or partial dilatation, sometimes spoken of as distension of the stomach, which is quite a different condition-we find only one portion of the stomach dilated, the cardiac end. That this form is a true dilatation and not a distension is shown by its liability to persist as

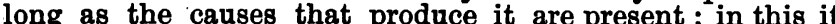
resembles dilatation of the cavities of the heart. In this variety the dislocation is upwards, by which I mean the yielding of the stomach wall at this portion causes it to press upwards against the diaphragm, or upwards and to the left in front of the spleen, where it does not meet with the same resistance as is offered by the diaphragm. When the latter is in a lax condition the dilated stomach presses directly upwards against the heart, producing a sense of uneasiness in the cardiac region, pain in left side, and occasional attacks of syncope.

The first variety, or that of complete dilatation, is mainly associated with organic disease of the stomach itself, accompanied by obstruction at the pyloric end. Obstruction giving rise to this aggravated form of dilatation is traceable to conditions in the pylorus itself, or the walls of the stomach in its vicinity, or to conditions outside of them. As a rule, it may be broadly stated that persistent dilatation in an aggravated form is always due to lesions within the stomach cavity. The causes acting from within the stomach leading to obstruction of the pylorus in the order of their frequency according to my experience are (a) cicatrisation of simple ulcers in the neighbourhood of pylorus; $(b)$ malignant disease attacking the pylorus ; $(c)$ fibroid thickening of pylorus, the result of inflammatory changes ; $(d)$ inflammatory conditions of the mucous membrane of the stomach and pylorus leading to pyloric spasm; such is the condition found in fatal cases of what is called the acute dilatation of alcoholics; $(e)$ foreign bodies such as coins or plum stones retained in the stomach and forming sacculated diverticula in the neighbourhood of the pylorus; $(f)$ pedunculated tumours growing from the wall of the stomach and falling against the pyloric opening.

The causes arising outside the pyloric walls leading to obstruction and dilatation are (a) pressure on the pylorus and duodenum by an enlarged organ in its vicinity, such as we find in some forms of enlargement of the liver or gall bladder; (b) tumours in the fissure of the liver especially; (c) pressure on pylorus of peritoneal or retroperitoneal tumours ; $(d)$ floating kidney; $(e)$ cystic disease of kidney pressing forwaid; $(t)$ kinking of duodenum or pylorus from non-yielding of gastro-hepatic ligaments when stomach is dragged down by the weight of its own fluid contents when in a dilated condition.

If we direct our attention to the second form of dilatation which I have alluded to as partial, and which is the form most frequently met with in all functional gastric troubles, and consider the causes that give rise to it, we find these causes for the most part associated with derangements of digestion where from alteration in the gastric contents gases are generated which by their continued distending action lead to dilatation of the stomach cavity. But the most frequent cause of this partial dilatation in my experience is an atonic condition of the muscle wall of the stomach, and as the cardiac end is the most muscular portion, an atonic condition of this muscle is more likely to be followed by yielding of this particular end than towards the pyloric one, which is more fibrous in structure. This form of dilatation is consequently found in all adynamic conditions. It is present in all forms of anæmia, pernicious anæmia included, and is constantly present in chlorosis, but not where gastric ulcer is associated with that affection, as the condition of the stomach in gastric ulcer is the very opposite to that of dilatationnamely, one of irritable contraction of its muscle from the irritation produced by the ulcer and the excess of $\mathrm{HCl}$. present with it. When dilatation of the cardiac end is present in association with gastric ulcer, hour-glass contraction of the stomach is usually found.

You find this form of dilatation in nearly all cases of advanced phthisis, also as a consequence of long-continued illnesses with pyrexia, such as in typhoid fever, being frequently present during the convalescent period following that affection. You find it accompanying the exhaustion of long-continued suppuration either in bones or elsewhere, also after many of the exanthemata and after influenza. In children such form of stomach dilatation follows subacute gastritis or gastro-enteritis, but dilatation is rare in children except from this cause. We meet with it in that important form of degeneration of muscles characterised by fatty changes, and also in chronic alcoholism, which is frequently accompanied by such changes. Acute dilatation due to paralysis of the essential nerves of the stomach, the pneumogastrics, should be here mentioned, but it more properly belongs to the graver forms of dilatation.

Having enumerated the causes that lead to both forms of dilatation, I will briefly allude to the symptoms characteristic of each.

In the first form, where there is aggravated dilatation, and where sooner or later the stomach is dislocated downwards, the symptoms of this condition are most characteristic. Prominent amongst these symptoms is vomiting. This vomiting differs in the time at which it occurs and in its character from the vomiting that takes place in other affections of the stomach; it does not take place after a meal, nor does it come on once a day, or twice a day, but generally every other day, and frequently takes place only at night (a most characteristic symptom of dilatation), or the intervals between attacks of vomiting may be extended to days, or even to a week or longer.

In other forms of stomach affection where vomiting occurs it is usually the result of some article of diet that disagrees, or some inflammatory or irritable condition of the stomach produced by food, and the vomiting as a rule follows the ingestion of such food; such is the condition of things in gastric ulcer and acute and subacute gastritis. Though patients with dilated stomachs do not suffer from vomiting after food, some hours after food is is taken they complain of pain, heartburn, and gaseous eructations, which are repeated after each meal, and are ultimately only relieved by vomiting. When the intervals between the acts of vomiting are counted by days, the stomach, in my experience, is usually found dislocated, and very much dilated.

In no other gastric affection accompanied by vomiting is the vomited matter so acid or so large in quantity as in cases of dilatation. Patients themselves will express the opinion that they vomit more than they eat or drink. The vomited matter is, as a rule, frothy, and, when allowed to stand for some time, bubbles will appear on the surface due to fermentation and the evolution of gases.

If we examine it chemically and by the aid of the micro- 
scope, it will be found that the acidity is rarely due to hydrochloric acid, but to the volatile or organic fatty acids-lactic, butyric, or acetic. Microscopically, we find great numbers of fungl associated with fermentation sarcina, when the liquid contents are retained for a considerable period, say a number of days; torula, when the intervals between the attacks of vomiting are shorter. We find also bacteria in great numbers. During normal digestion bacteria are never found, nor are they likely to exist in healthy gastric contents, more especlally the hurtful organisms which are destroyed by the antiseptic action of the hydrochloric acid in the gastric secretion. In aggravated cases of dilatation this acid is found in only small quantity, not sufficient to kill off bacterial growths, which continue to grow and generate toxins.

The most salient and characteristic physical sign of dilatation is splash on succussion of the stomach. It is not easy in all cases to ascertain the presence of this peculiar splash. Mere jogging of the stomach alone in one direction or another will not always determine its presence, and especially if this mancuvre is confined to the epigastric region alone. When dislocation has taken place, the entire abdominal cavity has to be searched before this symptom is elicited, even though dilatation to a formidable extent is present. It is also absent in many cases, owing to the stomach being emptied by vomiting previously, and after lavage by the stomach tube, as a considerable quantity of fluid and air must be present for its production. To nothing can I better compare this pecullar splash than the sound produced in an incompletely filled water bed by jogging or shaking it, or to the splash produced in a water tank by agitation. It has come to be known by the students of our hospital as the water-tank sound of dilated stomach.

Another constant symptom of the graver form of dilatation is obstinate constipation, the bowels often not moving without the aid of purgatives for days or even weeks at a time, and then only imperfectly, the evacuations being dry and lumpy, and lodgments in the colon being not an infrequent accompaniment. This condition always indicates a very considerable narrowing of the pylorus, through which the stomach sontents are not passing; and the intestines, losing the stimulus the stomach contents afford, cease to secrete, and pass themselves into an atonic condition. If with this dryness of the bowel vomiting of all stomach contents is constantly taking place, a desiccation of the tissues ensues, and some authorities consider a fatal termination results from this cause more than from any other.

Tetany extending from the muscles of the abdomen to the extremities and muscles of the neck and back often accompanies this condition, and I have seen it prove fatal. This tetany would seem to be due not so much to this desiccation as to the absorption of some toxin from the stomach contents. Another symptom associated with dilatation and generally indicative of considerable obstruction at the pylorus is visible contraction of the stomach seen through the abdominal wall. The contractions generally travel from left to right, and may be excited by pinching or stroking over the stomach. They are due to hypertrophy of the muscle wall of the stomach in trying to force its contents through the narrowed opening of the pylorus, such hypertrophy being similar to what occurs in the left ventricle of the heart in aortic obstruction. Cramps in the fingers are frequently complained of by patients the subjects of dilatation, and nodular growths on some of the joints of the phalanges are present in sgreat many cases, as pointed out by Bouchard. Coldness of the feet and hands, and redness of the nose, acne of the face and back, and attacks of urticaria are frequent symptoms. We sometimes find evidences of vasomotor disturbances such as sweating of the face and body, often of one side only, and accompanied by a peculiar pungent acid smell, probably due to the elimination of the fatty acids by the skin.

I will now direct your attention to the symptoms that are indieative of the second form of dilatation $\mathrm{I}$ have alluded to as that of partial dilatation, as this form of dilatation is found accompanying almost all the stomach affections known us dyspepsias. It is constantly present in that form of functional derangement known as catarrh of the stomach, an affection which may arise from ordinary cold catching, as a patient may get a cold in the stomach as readily as in the head or throat. It is also found in the gastric catarrh associated with cirrhosis of the liver and with valvular lesions of the heart where the liver is enlarged. Gastric catarrh, however, owes its origin more frequently to ingestion of unsuitable food or liquid, which, when retained too long in the stomach (the gastric secretion being defective) undergoes fermentative changes, such, for instance, as the catarrhal dyspepsia which is so common an affection in those who live constantly on tea and uncooked starch foods.

Here not only the quantity of the liquid taken but the character of the food is favourable to fermentation and the formation of the fatty acids. We find as articles of diet amongst the poor bread unsuitably baked or other badlycooked starchy food, such as potatoes, which are very prone to undergo fermentative changes leading to the formation of acids, especially acetic acid. Amongst the poor, too, there is a great tendeney when dyspepsia is present to seek relief by means of alcohol in some form. The alcohol taken under these conditions is liable to contribute still more to the acetic acid fermentation, and to cause, with lactic and butyric acids, the acid sensation and heartburn such patients so frequently complain of.

You will always find the minor form of dilatation present in such cases if you look for it, and the splash characteristic of it in the left epigastric and hypochondriac regions. In a former portion of $\mathrm{my}$ paper I alluded to the frequency with which this minor form of dilatation is met with in all atonic conditions. In some cases it would seem that an intrinsic atonic condition of the gastric muscle just as frequently results from the poisons that are absorbed into it during defective digestion and directly paralyse its functions. Turck has quite recently investigated the toxins of the stomach in cases of gastritis associated with dilatation, and found that the filtered contents injected into rabbits produced paralysis in an hour and a-half, and in larger doses killed. A reference to his paper will be found in the New York Medical Journal of February 22nd, 1896 .

Constipation in patients the subjects of the minor form of dilatation is a very frequent symptom, but not to the same extent as in the graver form, and vomiting as a rule is not present, the stomach contents ultimately finding their way into the intestines, by which they are absorbed with the toxins contained in them, and which produce the vague nervous symptoms such patients complain of. Amongst these symptoms hypochondriasis is a common one; weariness on the slightest exertion and a disinclination for exertion; headache, pain in the left side, and palpitation of the heart, and transient or intermitting anæmia are frequently present also. You also find symptoms referable to the lungs, throat, or bronchi in dilatation of the 'stomach where the contents are for some time retained, especially in the more aggravated form of the affection.

I have lately had under my care in the Mater Misericordiæ Hospital a patient with dilatation, who, while undergoing some preliminary examination of his stomach contents preparatory to lavement, got a severe attack of spasmodic asthma to which he was not previously liable; he had not vomited for days previously, and succussion showed the stomach dilated and filled with fermenting contents. In the middle of the night, without being exposed to cold in any way, he got an attack of asthma, with considerable dyspnoea and bronchial obstruction, which persisted until his stomach was washed out, when it gradually subsided. That his attack of asthma was due to some toxin absorbed from his stomach I think there could have been no doubt. What the nature of that toxin was I am unable to say; possibly it may have been due to the fatty acids, butyric or lactic acid being eliminated by the bronchial mucous membrane. They are principally eliminated by the skin, but also by the-lungs, and it is possible the attack of asthma in this case may have been due to their elimination by this channel.

We know other toxins in the blood produce asthma, uræmia, and gout, for instance, and we know that many of the toxins formed in the stomach affect the individual in various ways, some of them causing spasm and cramps, and others, as in Turck's experiments, causing paralysis and neuritis. The most reliable symptom indicative of the partial form of dilatation, as it is of the graver form, is splash on succussion. This splash in cases of left-sided dilatation is invariably found when the stomach contents are delayed and 
fermenting, and is never found in a healthy stomach no matter what the amount or what the character of its contents. It can always be obtained by succussion of the stomach in the left epigastric region in an upward and outward direction in a line between the umbilicus and the left costal cartilages: When the stomach is dilated but empty a tympanitic note takes its place, with this difference, that the tympanitic note is heard over a large area of both the left epigastric and hypochondriac regions, and extending from here upwards to the left nipple and outwards from this level to the midaxillary line. Under few circumstances is a tympanic note heard in this situation differing from that of ordinary thoracic resonance except in dilatation of this end of the stomach. It is consequently always as reliable of thls condition as the splash sound, and both together are conclusive.

Our methods of diagnosis at the Mater Misericordiæ are : If in an hour or two after a test meal splash is elicited or splash and a tympanic note extending to or near the nipple line dilatation is diagnosed. If the splash is heard at the end of five hours or longer an atonic condition of the muscle wall of the stomach is assumed to exist. To ascertain whether the dilatation is due to fermenting contents or atony alone we siphon off the contents at different periods after the test meal, and examine it chemically. If lactic acid persists for some hours after the test meal, hydrochloric acid being absent or present in only small quantities, lactic acid fermentation is present; if it is constantly present with hydrochloric altogether absent, cancer of stomach or pylorus is usually diagnosed.

If butyric, latic, and acetic acids are found there is no doubt fermentation of food contents is going on. If the solvent power of the pepsine present is feeble in its action on proteids, or produces no solvent action on them, secretion is defective or atrophy of gastric glands is diagnosed. For the determination of the existence of hydrochloric acid either Toepfer's test (dimethyl-amido-azobenzol) the benzopurpurin, or Gunzburg's phloroglucin and vanilin tests are used. For the detection of lactic acid a watery solution of perchloride of iron is used which is a very certain test. Butyric and acetic acids are detected by their smell when the filtered gastric contents are treated by ether, which extracts them out.

The chemical test for an atonic condition of the muscle wall of the stomach (the pylorus being pervious) is the administration of salol, which is unchanged by the stomach but splits up into its component elements phenol and salicylic acid when it reaches the intestines, the salicylic acid being secreted by the kidneys and appearing in the urine as salacyluric acid.

If this acid is not found in the urine until hours after the administration of the salol, an atonic condition of the muscle wall of the stomach is assumed to exist. For the extraction af the contents of the stomach we find the ordinary siphon india-rubber tube, such as is used for washing out purposes is quite sufficient. When passed into the stomach and allowed to remain for a few minutes the part projecting in front of the teeth is throttled, and held so until withdrawn, a drachm or two of the contents of the stomach is found in the end of the tube near its point, which is found quite sufficient for all purposes of examination. The tube and funnel which I use for both extraction and washing out has a stopcock fitted to it, which insures all air being shat out in the process of extraction.

The title of my paper did not include a description of the methods of treatment in cases of dilatation, bat I do not wish to dismiss the subject without a few words on that allimportant subject. For cases of the graver form of dilatation associated with obstruction at the pylorus, whether arising from or within the stomach, a suitable pylorectomy or gastroenterostomy is the only torm of treatment likely to produce a cure or to procure amelioration of the distressing conditions present, and when contractions and cramp of the stomach or symptoms of tetany arise operation should be urged at once.

The only other alternative to procure temporary rellef is washing out the stomach. I know of no other treatment in the graver form of dilatation which brings so much comfort to our patient, and even in the lesser form of dilatation I have no hesitation in resorting to it' when catarrh of the stomach with fermentation and acidity distress the patient. It is not necessary I should describe the methods of its performance, but a few words as to the best time for doing it may not be out of place. Lavage of the stomach is always best done in the morning, before food is taken, as by that time the food of the previous day, if not digested normally, is at all events liquefied sufficiently, either by fermentation or by growing colonies of bacteria, to prevent its blocking the tube in the process of extraction. Having ascertained the size to which the stomach is dilated, from a pint to two pints of hot water is first injected through the tube, and then siphoned back so as to thorougly wash the cavity. Next, a pint of some alkaline solution is introduced without withdrawing the tube, and allowed to remain for a few minutes (while the stomach is agitated between the fingers) and then siphoned back and the tube withdrawn.

The selection of the solution is not a matter of much importance; lime water answers very well, or a few teaspoonfuls of bicarbonate of soda or potass of lithia dissolved in a pint of hot water, or if the breath is fœtid, some boric acid, peroxide of hydrogen, or Sanitas may be substituted.

Turck has lately introduced a novelty in the shape of an instrument for gastric dilatation which he has called a gyromele. This instrument consists of a flexible cable to the end of which is attached a spiral spring covered with a sponge. To determine the outlines of the dilated stomach the tube is introduced into the organ and the apparatus set in motion. The revolving sponge can be easily palpated through the abdominal wall along the entire length of the stomach. The gyromele can be used as a therapeutic agent as well. In chronic catarrh of the stomach the revolutions of the sponge are extremely useful in effecting the removal of mucus from the surface of the mucous membrane, while in atonic conditions of the organ the friction thus applied to its inner surface acts as a stimulant to the muscular tissue. Weak solutions of nitrate of silver on the sponge have been recommended as an application to the mucous membrane in this manner. Massage of the stomach is also a most important method of treatment in the atonic variety of dilatation. Electricity I have tried, but I am disappointed with it, and rely more on careful massage to effect good results.

To check fermentation I generally prescribe creasote or carbonate of guaiacol combined with bismuth, or sulphurous acid, or the hyposulphites just as I find them to agree best, and where pain is present iodoform, carbolic acid, or ichthyol in pill combined with opium or Dover's powder.

The minor form of dilatation is generally very amenable to treatment, careful regulation of the diet being in many cases the principal treatment necessary where injudicious dietary leading to fermentation is the cardse. When a certain amount of catarrh is associated with this I find the alkaline carbonates, especially the bicarbonate of soda with nux vomica, and a vegetable bitter taken before food, very efficacious; it has the double property of dissolving mucus and exciting a more healthy acid secretion afterwards, besides lessening the tendency to acid fermentation. The addition of bismuth to such a combination is also very comforting if there is much gastralgia or heartburn present. If after this treatment for some days splash is still elicited four or five hours after a meal, an atonic condition of the gastric muscle is probably present, and I then give in addition four or five drops of liquor strychninæ after each meal. I consider no other drug to compare with it when a lax and atonic condition of the stomach wall existe. Purgatives given at the same time have a most wonderful influence in emptying a full dilated stomach : the peristalsis of the intestine excited by them seems to be communicated to the gastric wall reversely and to excite its contractions. Where catarrh is chronic and constantly leading to dilatation occasional lavage, combined with the above treat ment, will generally effect a cure. Pepsine, though often prescribed in such cases, I have little faith in as a means of treatment; it is at best but temporising, and its administration seems to me both unscientific and useless; in my experience it often sickens the patient, and increases the dilatation present, and certainly does not lessen the fermentation.

In cases of atrophy of the gastric glands and of marasmus in advanced cancer of the stomach walls when combined with hydrochloric acid, it assists the patients to digest proteids, but it is not as efficacious with a mixed dict in this respect as many of the malt extracts. 
A consideration of the various kinds of food suitable for the different conditions that lead to dilatation $I$ have not touched on, and I have contented .myself with calling atter.tion to the more obvious methods of treatment I have usually found efficacious.

\section{WIDAL'S SERO-DIAGNOSIS OF TYPHOID FEVER.*}

Bx E. T. FISON, M.B., B.C.Cantab ,

(From the Bacteriological Laboratory of St. George's Hospital.)

\section{INTRODUCTORY}

IN the practice of medicine certain and early diagnosis is the most important factor. To Widal belongs the honour of having shown that it is possible with some certainty to diagnose enteric fever by a method he terms "sero-diagnosis." I propose to give a cursory history of the evolution of this method. As long as eight years ago work directly bearing upon this subject was published, since then much more has been learned; but it was not until last year that these observations were turned to practical use.

In 1889 Charrin and Roger, while working at the cause of immunity, found that sera from animals, which had been injected with living or dead cultures of bacillus pyocyaneus, exerted an effect on the living bacillus pyocyaneus; they proved this by growing bacillus pyocyaneus in a test tube in serum from an animal immunised against it; the bacill formed flakes and fell to the bottom of the tube, whereas the supernatant fluid became clear. If the bacillus pyocyaneus were grown in serum from a non-immunised animal uniform turbidity resulted.

In 1894 Issaeff and Ivanoff made a like observation on a vibrio described by Ivanoff.

In $1894 \mathrm{R}$. Pfeiffer injected into the peritoneal cavity of a guinea-pig, previously immunised against spirillum choleræ, a culture of living cholera microbes. He found on examining the peritoneal exudation that the spirilla had lost their motility and had come to rest in clumps, and also that after a time they underwent a granular degeneration and ultimately disappeared.

He also found the same phenomena happened with bacillus typhosus. If bacilli not of the same species as the one which had been used to immunise the animal were injected, clumping did not take place.

He also showed that the phenomena of degeneration and ultimate absorption of the microbes occurred when a living typhoid culture was mixed with serum of an animal im munised against typhoid and injected into the peritoneal cavity of a non-immunised animal. This came to be used as a test for doubtful cultures of bacillus typhosus.

The clumping, granular degeneration, and ultimate absorption of the microbes observed when specific living organisms were injected into the peritoneal cavity of an animal immunised against that organism, as a whole, came to be known as "Pfeiffer's Reaction"

Metchnikoff and Bordet next showed that the phenomena, which Pfeiffer observed in the living animal, could be seen in the hanging drop preparation under the microscope. To apply this test one drop of serum of an animal immunised against the specific microbe is added to two drops of an emulsion of the donbtful calture; if the microbe be the specific one the organisms come to rest in clumps, and if more serum be added granular degeneration takes place. Vibrios or bacilli other than the one against which the animal is immunised do not react in this way. Gruber and Durham mixed in a test tube the microbe to be tested with a more or less diluted serum from an animal immunised against the microbe to be found. If the microbe were the specific one, the organisms in half to one hour fell to the bottom of the test tube and the supernatant fluid became clear; if the microbe belonged to $a$ species different from the one against which the animal has been immunised, the contents of the tube remained uniform.

The above experiments showed that the serum from an animal in a state of immunity exhibited definite qualities when mixed with the specific organism only. The changes which resulted were also used as a test for doubtful

\footnotetext{
* This is part of a Thesis written for the M.D. degree of Cambridge
}

microbes. Hitherto these changes in the organisms had only been shown with sera from animals in the stage of immunity.

Widal, however, believed that the blood of patients suffering from typhoid fever acquired qualities which gave the changes mentioned above at a period long before immunity could have been reached, indeed during the period of infection. On this hypothesis, he thought it would be more practical to mix typhoid bacilli with serum from typhoid patients, and see whether the same changes observed by Pfeiffer in vivo or in vitro by Metchnikoff, Bordet, and Durham took place, and if so to make use of these phenomena for the diagnosis of typhoid fever rather than of typhoid bacilli.

This he did, and last June brought before the Medical Society of Paris a new method for the diagnosis of typhoid fever which he termed "sero-diagnosis." He described several processes. One consisted in adding a few drops of serum from a suspected typhoid case to a bouillon culture of Eberth's bacillus, already cloudy, and observing a few hours later whether it became clear at the top with a precipitate at the bottom of the tube. Another consisted in adding a few drops of serum from a supposed typhoid patient to a bouillon tube, then inoculating with Eberth's bacillus and observing whether the same phenomena took place as mentioned above. A third method was the mingling of one drop of serum from the supposed typhoid patient with ten drops of a young bouillon culture of bacillus typhosus on a cover glass, and then examining under the microscope to see whether the microbes formed heaps or agglomerations. $\mathrm{He}$ also showed that the reaction eould be obtained with dried serum or blood emulsified and then added to bacillus typhosus.

In fairness to Grünbaum, it must be stated that he had heen working at the same subject simultaneously with Widal: but his theses were not published till two months after Widal's announcement; he cannot, therefore, have the same claim as Widal to this most important discovery.

Chantemesse added typhoid organisms to sera from cases of pneumonia, influenza, erysipelas, and peritonitis, and found that no agglutination of the organisms took place.

Professor Wright, acting on an observation of Widal that serum from patients suffering from typhoid fever showed the agglutinative action when added to dead typhoid organisms in the same way as when mixed with living ones, published a paper on this point in the British Medical Journal of May I 5 th, I 897 .

He has experimented with the bacillus typhosus and the micrococcus melitensis of Bruce. Emulsions of the living organisms were taken up, placed in capsules, and then subjected to a temperature of $60^{\circ} \mathrm{C}$. for five to ten minutes: serum was then taken from a subject who had been vaccinated against typhoid fever siximonths previously, and from a patient suffering from Malta fever; these sera were added to the respective specific dead organisms in a sedimentation tube. He found that the reaction was quite as definite as when these sera were added to living organisms, and that there was no difference in the method of agglomeration.

If this new method be found reliable, it will greatly facilitate the performance of the test, and any practitioner who cares to provide himself with sterilised cultures of bacillus typhosus will be able to conduct it.

The serum of typhoid patients shows this agglatinative reaction early in the disease. Johnston and McTaggart state that they observed an incomplete reaction within forty-eight hours of the attack of the fever. Most observers are agreed that the serum obtains this agglutinating power by the end of the first week.

The serum from patients who have had typhoid fever seems to retain these qualities for a remarkable length of time, and may as a rule be reckoned by years. This fact bears strongly on immunity to second attacks of typhoid fever, for it is well known that two attacks of the disease in the same individual is a rare coincidence. Bestdes the serum of typhoid patients reacting in this way with typhoid bacilli, it is claimed that the milk of nursing women suffering from typhoid fever, the tears, the exadations from blisters, and the urine give the same reaction.

Achard states that the blood of a fotus from a mother in the acute stage of typhoid fever gave no reaction; so it 